\title{
Idiopathic Ventricular Tachycardia AsSociated With AV RECIPROCATING TACHYCARDIA
}

\author{
Charles-Lwanga K. Bennin, MD, Avinash Chandra, $M D$
}

\section{Case Report}

A forty-six year old female patient with a medical history of asthma and seasonal allergies presented with a two day history of shortness of breath, associated with palpitations. She denied chest pain or diaphoresis. Patient reported past history of similar complains that usually lasted less than two minutes and were relieved with aspirin. The longest episode of palpitations lasted approximately twenty minutes.

Her social history was significant for tobacco and alcohol abuse, but negative for substance abuse. Her family history was significant for cardiovascular disease.

Pertinent findings on physical examination were significant for elevated blood pressure at $156 \mathrm{mmHg} / 112 \mathrm{mmHg}$ and tachycardia at 112 beats per minute. Her electrocardiogram (EKG) showed tachycardia at a rate of 150 beats per minute with a long RP interval concerning for atypical AV reciprocating tachycardia (AVRT). The EKG also revealed right bundle branch block with a left axis deviation (Figure 1) and non-sustained ventricular tachycardia (Figure 2) indicating a diagnosis of idiopathic ventricular tachycardia. A urine drug screen was positive for cocaine.

\section{Discussion}

Idiopathic fascicular ventricular tachycardia has been reported in literature by Cohen in $1972^{1}$ and Zipes in $1979^{2}$. These electro- physiologic findings are unique in that the QRS complexes are narrow, especially when compared to the typical wide QRS complex ventricular tachycardia and a right bundle branch morphology. The fascicle activated on re-entry determines the axis - left axis deviation (left posterior fascicle) and right axis deviation (left anterior fascicle). Some case have been reported of familial presentations of idiopathic ventricular tachycardia. ${ }^{3}$

Eighty percent $(80 \%)$ of idiopathic ventricular tachycardia originates from the ventricular out flow tracts or the coronary cusp. ${ }^{4}$ The origin of these arrhythmias has been attributed to the posterior inferior left ventricle in a region of the left posterior fascicle. ${ }^{5}$ This region probably has a high degree of reentrant or triggered automicity. ${ }^{6}$ The adjacent posterior left bundle branch may or may not be involved in the anterograde limb of the reentrant circuit.?

Idiopathic ventricular tachycardia, having a morphology of left bundle branch block and right axis deviation, is believed to originate in the right ventricular outflow tract, but may arise from the right ventricular outflow tract in about $18 \%$ of cases. ${ }^{8}$ According to Francis et al. idiopathic fascicular ventricular tachycardia has been limited to three subtypes in order of prevalence; left posterior fascicular ventricular tachycardia with a right bundle branch block morphology and superior axis configuration; left anterior fascicular ventricular tachycardia with right bundle branch block and right-axis

Figure 1. EKG on admission showing right bundle branch block with a left axis deviation.

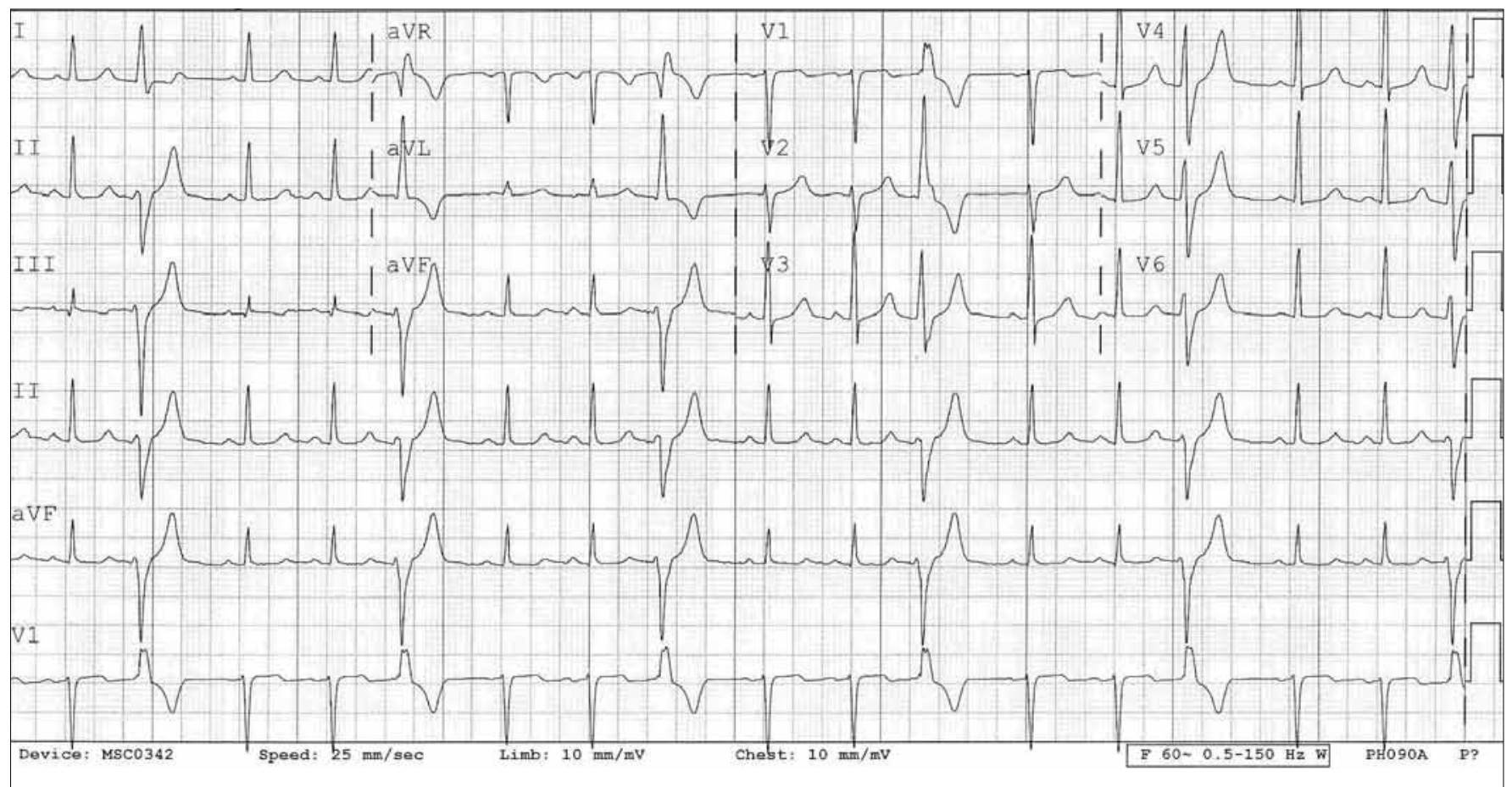


Figure 2. EKG on admission showing non-sustained ventricular tachycardia.

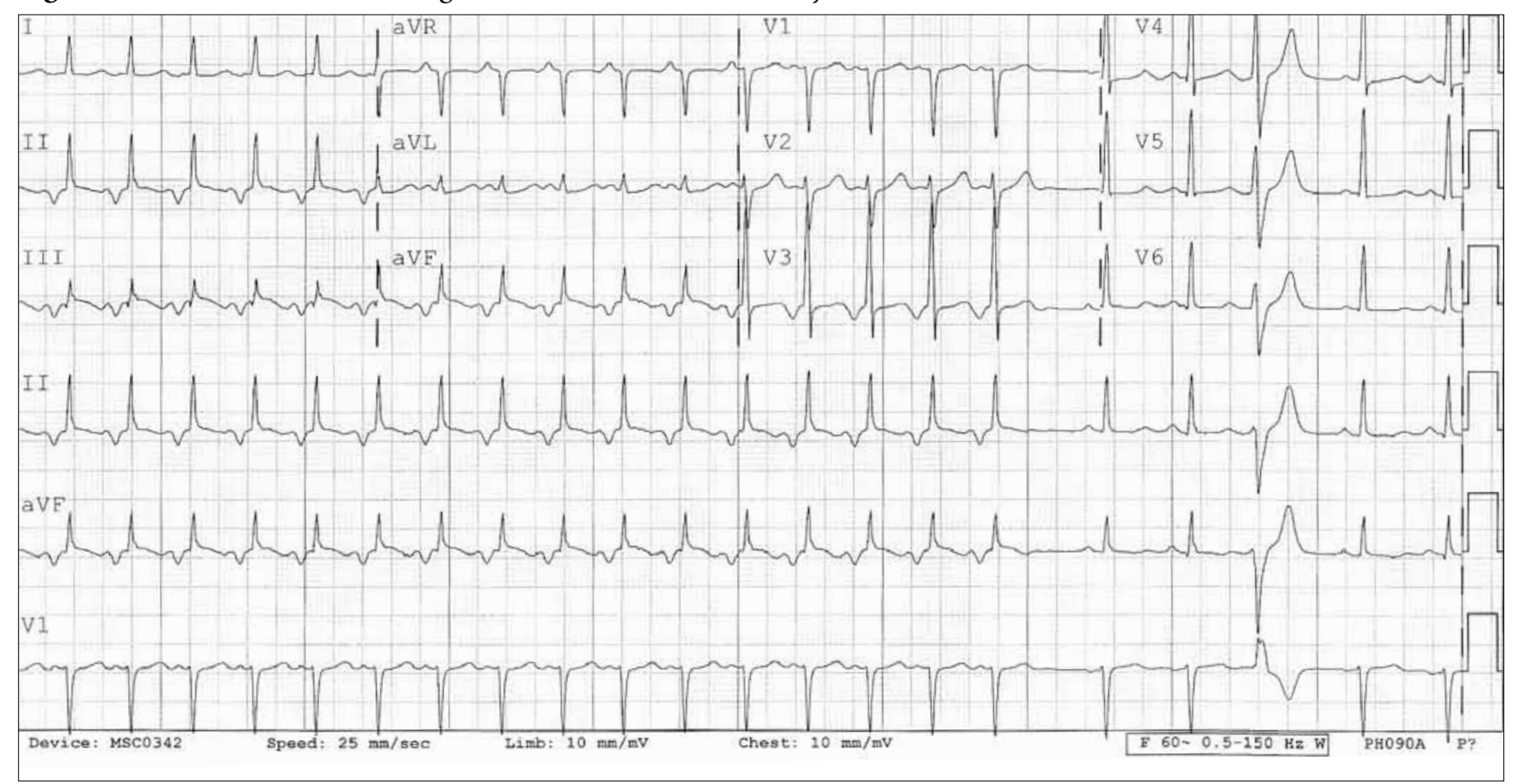

deviation configuration; and the rare presentation of upper septal fascicular ventricular tachycardia with a narrow QRS and normal axis configuration. ${ }^{6}$

Nogami also classified idiopathic ventricular tachycardia into adenosine sensitive, propranolol sensitive and verapamilsensitive fascicular ventricular tachycardia. ${ }^{9}$ It appears that fascicular ventricular tachycardia are sensitive to phenylalkylamine class L-Type calcium channel blockers such as verapamil as have been well described by Belhassen. ${ }^{10,11}$ Calcium channel blockers also suppress conduction through atrio-ventricular (AV) node, and are effective on both AV reciprocating tachycardia (AVRT) and AV nodal reentrant tachycardia (AVNRT).

Atrial pacing as well as supraventricular tachycardia has been shown to induce ventricular tachycardia due to either reentry or triggered automaticity. It is not uncommon to find co-existing tachyarrhythmia and a few cases have been reported of ventricular tachycardia initiated by atrial arrhythmias including AV reciprocating tachycardia also known as AV reentrant tachycardia (AVRT). ${ }^{5}$

Literature search using PubMed with the following keywords "Idiopathic ventricular tachycardia, AV reentrant tachycardia, AV reciprocating tachycardia, AVRT" produced 2 articles. One case series reported seven patients without structural heart disease in which AVNRT spontaneously triggered VT in three cases. ${ }^{12}$ Another case report illustrated a patient with Wolff-
Parkinson-White (WPW) syndrome. Ventricular tachycardia originating from the right ventricular outflow tract was induced during isoprenaline infusion. This also led to atrioventricular reentrant tachycardia (AVRT). This case report was significant in that the ventricular tachycardia was possibly driven by catecholamine stimulation ${ }^{13}$.

In our case report we present a patient with possible cocaine induced idiopathic ventricular tachycardia and AV reentrant tachycardia. Cocaine works on by increasing release of norepinephrine and dopamine and blocking reabsorption of norepinephrine, dopamine and serotonin. Cocaine also blocks sodium channels, thereby interfering with the propagation of action potentials. The release of catecholamines that occur in cocaine intake may exacerbate changes in the action potential threshold and hence stimulate automaticity. Management of idiopathic ventricular tachycardia includes radiofrequency ablation and intravenous verapamil.

\section{Reference}

1. Cohen HC, Gozo EG Jr, AP. Ventricular tachycardia with narrow QRS complexes (left posterior fascicular tachycardia). Circulation. 1972;45(5):1035-1043.

2. Zipes DP, Foster PR, Troup PJ, DH. P. Atrial induction of ventricular tachycardia: reentry versus triggered automaticity. Am J Cardiol. 1976;44(1):1-8.

3. Cho YK, Han SW, Kim YN. Cases of familial idiopathic left ventricular tachycardia. Europace. 2007;9(9):852-853.

4. Topilski I, Glick A, Belhassen B. Idiopathic left ventricular tachycardia with a right bundle branch block morphology and left axis deviation ("Belhassen type"): results of radiofrequency ablation in 18 patients. Isr Med Assoc J. 2004;6(4):195-200. 
5. Ichiro Watanabe, Satoshi Kunimoto, Kazuhiko Kondo, Toshiaki Kojima, Toshiko Nakai, Atsushi Shindo, Naohiro Oshikawa, Satoshi Saito, Yukio Ozawa, Kanmatsuse K. Radiofrequency Catheter Ablation of Coexistent Atrioventricular Reciprocating Tachycardia and Left Ventricular Tachycardia Originating in the Left Anterior Fascicle. Jpn Circ J 1999;63:223 -227.

6. Francis J, Venugopal K, Khadar SA, Sudhayakumar N, Gupta AK. Idiopathic fascicular ventricular tachycardia. Indian Pacing Electrophysiol J. 2004;4(3):98-103.

7. Kuo JY, Tai CT, Chiang CE, Yu WC, Huang JL, Hsieh MH, Hou CJ, Tsai CH, Ding YA, SA. C. Is the fascicle of left bundle branch involved in the reentrant circuit of verapamil-sensitive idiopathic left ventricular tachycardia? Pacing Clin Electrophysiol. . 2003;26 (10):1986-1992.

8. Belhassen B, Glick A, Herz I, Berger M, Swissa M. ECG of the month. Idiopathic ventricular tachycardia with a left bundle branch block morphology and right axis deviation. Isr Med Assoc J. 2003;5(9):679-680.
9. Nogami A. Idiopathic left ventricular tachycardia: assessment and treatment Card Electrophysiol Rev. 2002;6(4):448-457.

10. Belhassen B, Shapira I, Pelleg A, Copperman I, Kauli N, Laniado S. Idiopathic recurrent sustained ventricular tachycardia responsive to verapamil: an ECG-electrophysiologic entity. Am Heart J. 1984;108(4 Pt 1):1034-1037.

11. Belhassen B, Horowitz LN. Use of intravenous verapamil for ventricular tachycardia. Am J Cardiol. 1984;54(8):1131-1133.

12. Kautzner J, Cihák R, Vancura V, J. B. Coincidence of idiopathic ventricula outflow tract tachycardia and atrioventricular nodal reentrant tachycardia. Europace. 2003 5(3):215-220.

13. Washizuka T, Niwano S, Tsuchida K, Y. A. AV reentrant and idiopathic ventricular double tachycardias: complicated interactions between two tachycardias. Heart. 1999 81(3):318-320.

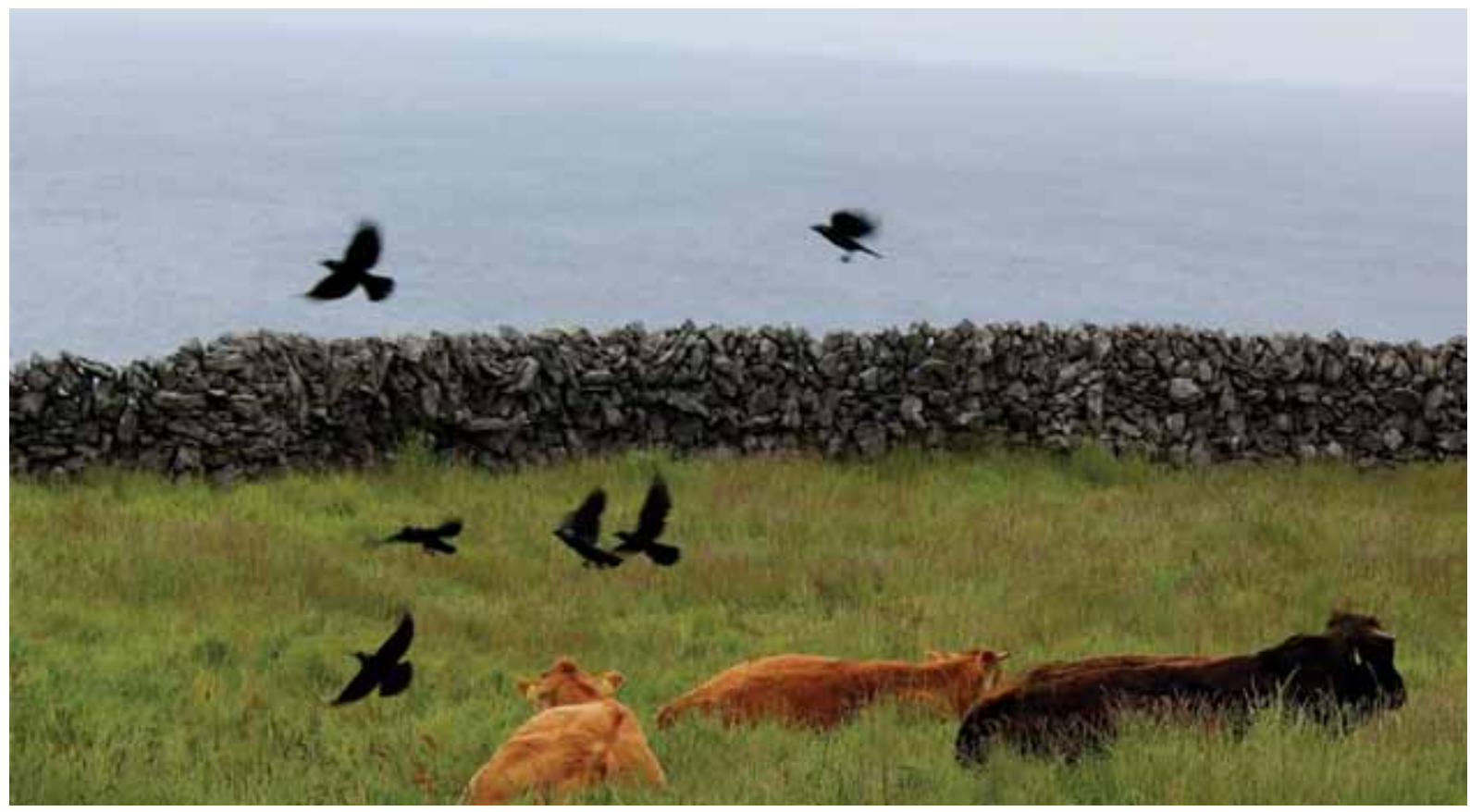

Photograph by Cecilia Kelly, MD 